\title{
Gender Recognition from Faces Using Bandlet and Local Binary Patterns
}

\author{
Faten A. Alomar, Ghulam Muhammad, Hatim \\ Aboalsamh, Muhammad Hussain, Anwar M. Mirza, \\ College of Computer and Information Sciences \\ King Saud University, Riyadh, Saudi Arabia \\ \{ghulam, faomar, mhussain, ammirza\}@ksu.edu.sa
}

\author{
George Bebis \\ Department of Computer Science and Engineering \\ University of Nevada at Reno, USA \\ bebis@cse.unr.edu
}

\begin{abstract}
In this paper, multi-scale bandlet and local binary pattern (LBP) based method for gender recognition from faces is proposed. Bandlet is one of the multi-resolution techniques that can adapt the orientation of the edges of the face images, and thereby can better capture the texture of a face image. After extracting bandlet coefficients from face images at different scales, LBP is applied to create a histogram, which is used as the feature to a minimum distance classifier. The experiments are performed using FERET grayscale face database, and the highest accuracy of $99.13 \%$ is obtained with the proposed method.
\end{abstract}

Keywords-Gender recognition; face images; FERET; bandlet

\section{INTRODUCTION}

The current research on face recognition involves developing accurate and robust algorithms against some inevitable constraints, as well as algorithms to recognize faces with respect to age, gender and race in order to enhance face recognition performance. The latter algorithms refer to "category specific" recognition systems, which are subsystems of a face recognition system [1]. Many research show that performing gender recognition prior face recognition as a preprocessing step is very us eful to decrease the computational burden and the speed of the system, especially when a database is large. Furthermore, they found that face recognition accuracy is increased when separate face recognizers are trained for gender $[2,3]$. Recognizing gender itself has some applications, such as gender-specific commercial advertis ements.

Many methods are proposed in the literature for gender recognition from faces. In [2], two databases, namely FERET [4] and a collected set of faces from www, are used with and without hair for gender recognition. Block-based local binary pattern (LBP) [1] and support vector machine (SVM) are used for the experiments. It was found that FERET images have higher accuracy than www images, and normalized faces without hair are more efficient than with hair faces. The best accuracy was found $92 \%$. Fang and Wang reduce the dimension of LBP features by splitting the neighbors into cross neighbors and diagonal neighbors, and thereby speed up the gender recognition system [5]. In FERET database, they achieve $92 \%$ accuracy. Weber local descriptor (WLD) based gender recognition is proposed in [6]. In WLD, differential excitation and gradient orientation are combined to form a robust texture descriptor [7]. In [6], block-based WLD histograms are concatenated to produce the feature vector set, and evaluated on the FERET database, which yielded $99.08 \%$ accuracy. Discrete wavelets transform (DWT), and principal component analysis (PCA) based gender recognition is proposed in [7]. First, an image is decomposed into subbands using a 3-level DWT, and PCA is applied on these subbands. In the classification step, Fisher linear discrimination is used. In FERET database, the accuracy of $93 \%$ is obtained by this method.

There are other methods that are applied in different databases. For example, a new local texture approach called interlaced derivative pattern (IDP) is proposed for gender recognition, and is evaluated in FRGC database [8]. The best result of $91.2 \%$ is achieved with 262 male and 195 female faces. Bui et al. introduced a combination of principal components from the global face, gradient face, and component (eye, nose, and mouth) face [9]. Using SVM classifier, the method obtained $98.2 \%$ accuracy with ORL database and 98.4\% with CalTech database.

Multi-resolution techniques are comparatively less used in the previous work for gender recognition. In this paper, bandlet, which is a multi-resolution technique, together with LBP is proposed for gender recognition. First, input normalized face image is decomposed into subbands of several scales. Each subband is then divided into blocks, and LBP histogram is extracted from each block of the subband. The histograms are concatenated to form a feature vector of the image. Optional feature selection algorithms in the form of the Fisher discrimination ratio (FDR) is applied to reduce the dimension of feature vector. Minimum distance classifier is used to classify male and female faces. The proposed method is evaluated on FERET grayscale face image database.

The rest of the paper is organized as follows. Section 2 describes the proposed method; Section 3 gives experimental results with discussion, and Section 4 draws some conclusion.

\section{PROPOSED METHOD}

Fig.1 shows a block diagram of the proposed gender recognition method. In the proposed method, bandlet and LBP are used to extract features, and minimum distance classifier is used to classify the gender. In the following subsections, each component is described in some details. 


\section{A. Bandlet Transform}

Image modalities consist of many geometric structures that carry perceptual information, and geometrical structures can improve image representation. A wavelet transformation technique does not take care of a geometric shape in an image region to represent sharp image transitions, while bandlet does. Representing sharp image transitions such as edges are expensive, but it is very efficient in removing noise, compressing the image, and to analyze texture and edges. The goal of bandlet is to take advantage of sharp image transitions (geometric structures) by calculating the geometric flow to form bandlet bases, which show directions of regular variations of image gray level [10]. To apply bandlet transform, the image is divided into square blocks, where each block or region $(\Omega i)$ includes at most one contour. If a block does not contain any contour, it implies that the image intensity is uniformly regular in that block. Bandlet transform is computed by approximating these regions using the wavelet bas is in $\mathrm{L}^{2}(\Omega)$ doma in [11]:

$$
\left\{\begin{array}{l}
\emptyset_{j, m}(x)=\emptyset_{j, m 1}(x 1) \emptyset_{j, m 2}(x 2) \\
\psi_{j, m}^{H}(x)=\emptyset_{j, m 1}(x 1) \psi_{j, m 2}(x 2) \\
\psi_{j, m}^{V}(x)=\psi_{j, m 1}(x 1) \emptyset_{j, m 2}(x 2) \\
\psi_{j, m}^{D}(x)=\psi_{j, m 1}(x 1) \psi_{j, m 2}(x 2)
\end{array}\right\}(j, m 1, m 2) \in I_{\Omega}
$$

where, we know that the wavelet transform is calculated as a product of elementary orthogonal operators, wavelet $\Psi(\mathrm{t})$ and scaling function $\phi(\mathrm{t})$ and the dilation $j$ and translation $k$ of them produces

$$
\psi_{j, k}(t)=2^{\frac{-j}{2}} \psi\left(2^{-j} t-k\right), \phi_{j, k}(t)=2^{\frac{-j}{2}} \psi\left(2^{-j} t-k\right),
$$

$I_{\Omega}$ is the index set of the geometry of the region $\Omega, x$ is the location of a pixel in the image, $\emptyset_{j, m}(x)$ is the coarse scale (approximation) and $\psi_{j, m}{ }^{H}(x), \quad \psi_{j, m}{ }^{\mathrm{V}}(x), \quad \psi_{j, m}{ }^{D}(x), \quad(j \in L$, $\left.m=\left[m_{1}, m_{2}\right], \quad x=\left[x_{1}, x_{2}\right] \in L^{2}\right)$ denote, respectively, the high horizontal frequency coefficients, high vertical frequency coefficients and high diagonal frequency coefficients decomposed by the dis crete wavelet.

Next, to compute the geometric flow in a region $\Omega$, the bandlet orthonormal basis will replace the wavelet bas is in Eq. (1), as in Eq. (2):

$$
\left\{\begin{array}{l}
\emptyset_{j, m}(x)=\emptyset_{j, m 1}(x 1) \emptyset_{j, m 2}(x 2-c(x 1)) \\
\psi_{j, m}^{H}(x)=\emptyset_{j, m 1}(x 1) \psi_{j, m 2}(x 2-c(x 1)) \\
\psi_{j, m}^{V}(x)=\psi_{j, m 1}(x 1) \emptyset_{j, m 2}(x 2-c(x 1)) \\
\psi_{j, m}^{D}(x)=\psi_{j, m 1}(x 1) \psi_{j, m 2}(x 2-c(x 1))
\end{array}\right\},, l>j, m 1, m 2 .
$$

After inserting Bandlet in the warped wavelet bas is, we get Eq. (3),

$$
\left\{\begin{array}{l}
\emptyset_{j, m}(x)=\emptyset_{j, m 1}(x 1) \emptyset_{j, m 2}(x 2-c(x 1)) \\
\psi_{j, m}^{H}(x)=\emptyset_{j, m 1}(x 1) \psi_{j, m 2}(x 2-c(x 1)) \\
\psi_{j, m}^{V}(x)=\psi_{j, m 1}(x 1) \emptyset_{j, m 2}(x 2-c(x 1)) \\
\psi_{j, m}^{D}(x)=\psi_{j, m 1}(x 1) \psi_{j, m 2}(x 2-c(x 1))
\end{array}\right\}{ }_{(j, m 1, m 2) \in I_{\Omega},}
$$

where $c(x)$ is the flow line of the fix translation parameter $x 2$, and we define $c(x)$ as:

$$
c(x)=\int_{x_{\min }}^{x} c^{\prime}(u) d u
$$

Note that the size of image division affects the geometric flows direction. If the block size is small, bandlet can accurately describe edges of images. An illustration of this argument is shown in Fig.2. Using Eq. (3), a set of bandlet coefficients are found.

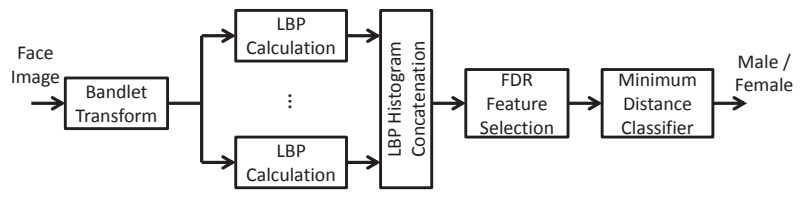

Fig. 1. Block diagram of the proposed method.
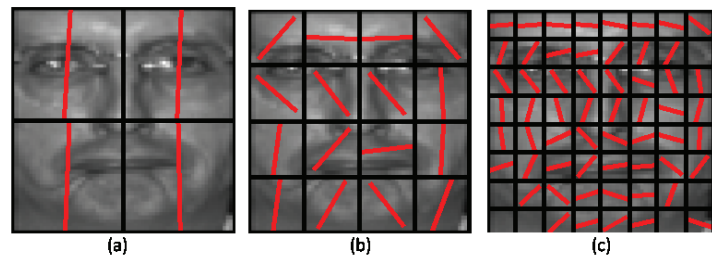

Fig. 2. Geometric flow representation using different block sizes: (a) big size, (b) medium size, (c) small size, of a normalized FERET grayscale face image.
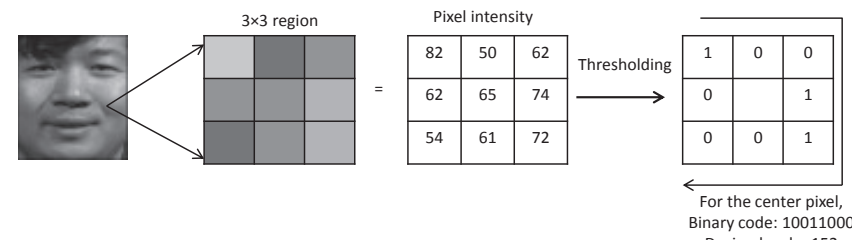

Fig. 3. The basic LBP operator.

\section{B. Local Binary Pattern (LBP)}

LBP is a popular local texture descriptor, which was first used to describe faces in [1]. It is an efficient technique due to its invariance to monotonic changes of gray level, computational efficiency, and highly discriminative power. It thresholds each $3 \times 3$ center pixel value with its neighborhood giving a binary number that has been assigned as a label to each pixel in the image (see Fig. 3). After that, it uses the histogram of those labels as a texture descriptor. In the case of different scales, LBP uses a circle of radius $R$, and $P$ is the number of local neighborhood on that circle (interpolated LBP) with various mappings. For detail description, readers may refer to [1].

\section{Feature Subset Selection}

Input image is first bandlet transformed into different scales to produce several subbands. Then each subband is divided into blocks and LBP histogram is calculated for each block. All the histograms from all the subbands are then concatenated to give a final feature vector for an image. However, this vector dimension is large, and hence, an optional feature selection algorithm is applied to reduce the dimension. In this paper, FDR is used as a feature selection criterion. The FDR is calculated per feature as the square of the difference between the means of both classes (in this case, male and female) over the summation of both classes' variances. 


\section{Minimum Distance Classifier}

In the proposed method, minimum distance classifier is adopted due to its simplicity. Four types of distance measures are investigated, which are city block distance (L1), Euclidean distance (L2), cosine distance, and chi-square distance (CS). The equations of these distance metrics are given below (Eq. 5 - 8). In these equations, $x$ and $y$ are the two classes, and $n$ is the number of features in each class.

$$
\begin{aligned}
& \text { L1: } d(X, Y)=\sum_{i=1}^{n}\left|x_{i}-y_{i}\right| \\
& \text { L2: } d(X, Y)=\sqrt{\sum_{i=1}^{n}\left(x_{i}-y_{i}\right)^{2}} \\
& \operatorname{COS:} d(X, Y)=-\cos (X, Y)=\frac{\sum_{i=1}^{n} x_{i} y_{i}}{\sqrt{\sum_{i=1}^{n} x_{i}^{2} \sum_{i=1}^{n} y_{i}^{2}}} \\
& \operatorname{CS:} d(X, Y)=\sum_{i=1}^{n} \frac{\left(x_{i}-y_{i}\right)^{2}}{x_{i}+y_{i}}
\end{aligned}
$$

\section{EXPERIMENT S AND DISCUSSION}

This section describes the database used, experimental setup, results, and provides discussion.

\section{A. Database}

In the experiments, FERET grayscale database is used. The face images are normalized to $64 \times 64$ resolution frontal faces with no hair. It consists of 994 different people's faces of which 403 are females and 591 are males. The 1204 face images of $f a$ set are used as training images of 746 are for males and 458 are for females, and 1196 face images of $f b$ set of which 740 are for males and 456 are for females are used as testing images.

\section{B. Experimental Setup}

Different sizes of blocks $(2 \times 2,4 \times 4,8 \times 8,16 \times 16$, and $32 \times 32)$ and various scales $(0,1$, and 2$)$ are used in bandlet transform. In the first experiment, only bandlet coefficients without LBP histogram are investigated. In the second experiment, the proposed method of bandlet and LBP is evaluated, while in the third experiment, the effect of FDR feature selection is examined.

\section{Results and Discussion}

Fig. 4 shows the effect of bandlet block sizes and scales on gender recognition. In all the experiments, L1 distance measure performs better than the three other distance measures. Therefore, all the results presented are with $\mathrm{L} 1$ distance measure. From Fig. 4(a), we find that the accuracies of block sizes $2 \times 2,4 \times 4$, and $8 \times 8$ are almost similar, while those for $16 \times 16$ and $32 \times 32$ are worse. Fig. 4(b) shows that the scale 2 has a bit higher accuracy than the scale 0 and 1 in the case of block size $2 \times 2$. The number of features in these cases is 4096 $(=64 \times 64)$.

Fig. 5 illustrates the effect of scale combination of bandlet with block size $2 \times 2$. Before combining the scales, the coefficients are normalized. Normalizing is done with the process of diving the bandlet coefficients vector, $\boldsymbol{a}$, by its norm $|\boldsymbol{a}|$ as $\boldsymbol{a}^{\wedge}=\boldsymbol{a} /|\boldsymbol{a}|$. The best accuracy of $97.3 \%$ is achieved with a combination of all the scales 0,1 , and 2 . It is noteworthy that normalized bandlet coefficients with scale 0 alone give $96.73 \%$ accuracy comparing to $94.54 \%$ with scale 0 without normalization.
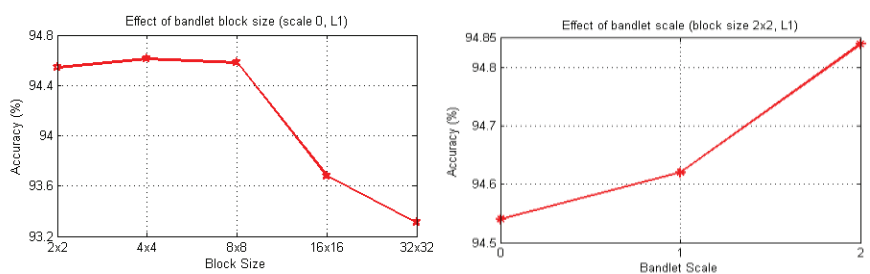

Fig. 4. Effects of (a) block size and (b) scale in bandlet on gender recognition.

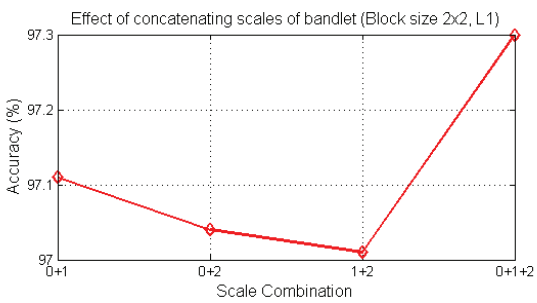

Fig. 5. Effect of combined scales in bandlet on gender recognition.

In the second type of experiments, the effect of LBP on bandlet coefficients is investigated. Using the bandlet, the image is decomposed into a number of scales. In the experiments, the execution of scale 0 bandlet coefficients is faster than higher scale. Therefore, LBP is applied on scale 0 normalized bandlet coefficients with block size $2 \times 2$ (bandlet block size, not the LBP block size). LBP histograms are obtained in blocks of bandlet image, and concatenated to give the final histogram, which is fed as a feature vector to the classifier. The basic LBP and the interpolated LBP with radius one and neighborhood of eight with different mappings are investigated. In the experiments, the basic LBP and the interpolated LBP with zero mapping perform better than other mappings (u2, ri, and riu2). Therefore, other mapping results are not reported in this paper.

Fig. 6 shows the gender recognition accuracies using normalized bandlet of scale 0 and LBP with different block sizes. The best average accuracy of $99.13 \%$ (male $98.9 \%$ and female $99.3 \%$ ) is achieved by the basic LBP and block size of $16 \times 8$, closely followed by $99.11 \%$ obtained by the interpolated LBP with zero mapping and block size of $16 \times 4$. The square block size $8 \times 8$ also has comparable performance. The whole image without dividing it into blocks performs the worst. The number of features in the best case is 8192 , which comes from 256 LBP bins multiplied by $32(=4 \times 8=64 / 16 \times 64 / 8)$ b locks . Average time per image needed in this best case is 0.028 seconds.

Applying LBP on the concatenated scales of normalized bandlet is also investigated. Though the number of features is 
increased in this experiment, the performance is not increased. For example, concatenating all the three scales of bandlet and applying the basic LBP on $16 \times 8$ block sizes yields 20480 features and $99.06 \%$ accuracy.

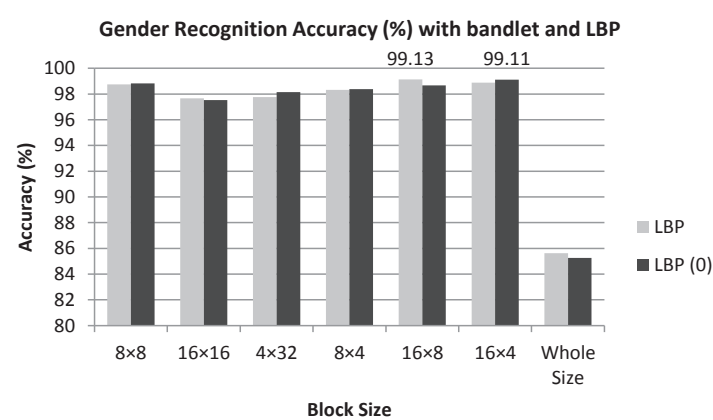

Fig. 6. Gender recognition accuracy (\%) using normalized bandlet scale 0 and LBP at different block sizes. LBP (0) represents zero mapping and LBP corresponds to the basic LBP.

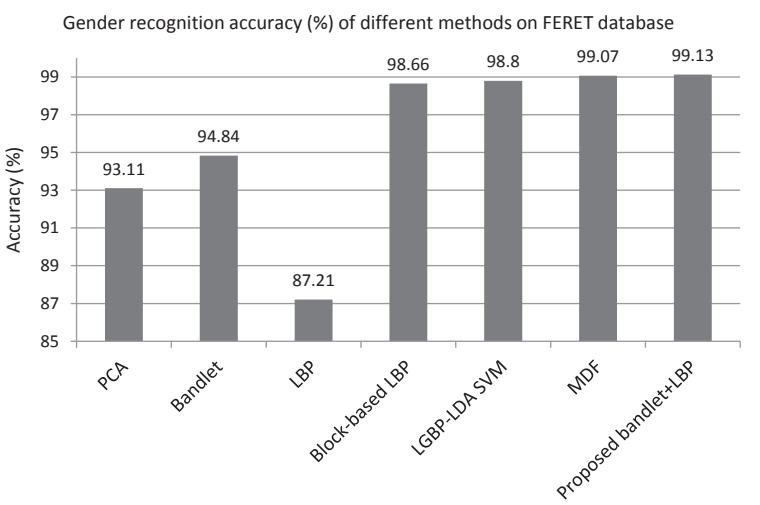

Fig. 7. Gender recognition accuracy (\%) of different methods on FERET database.

In the third type of experiment, FDR is applied on the features to reduce the dimension. FDR is calculated on the features of the best setup (99.13\%), which is normalized bandlet with scale 0 and $2 \times 2$ blocks with the basic LBP having block size of $16 \times 8$. In the experiment, the accuracy does not improve (remains at $99.13 \%$ ), however, the number of features is decreased from 8192 to 7673 .

\section{Comparison with Other Methods}

The proposed bandlet and LBP based method is compared with some other state of the art and conventional methods in FERET database for gender recognition. The compared methods are PCA, bandlet only, LBP only, block-based LBP (block size of $16 \times 8$ ), local Gabor binary pattern with linear discrimination analysis with SVM (LGBP-LDA SVM) [12], and multi-resolution decision fusion (MDF) method [13]. Fig. 7 shows the accuracies of the different methods. From the figure, it is found that the proposed method outperforms some of the conventional and recent methods. For examp le, the PCA and the LBP without blocks have the accuracies of $93.11 \%$ and $87.21 \%$, respectively, while LGBP-LDA SVM and MDF have better accuracies (98.8\% and $99.07 \%$, respectively). The proposed method has the highest accuracy of $99.13 \%$.

\section{CONCLUSION}

Multi-scale bandlet and LBP based method for gender recognition from face images is proposed. Bandlet coefficients are extracted at different scales from a face image, and LBP histogram is calculated from the coefficients at various blocks. The proposed method is evaluated in FERET gray face image database and achieves $99.13 \%$ accuracy, which is better than that of some state-of-the-art methods. However, the proposed method has a high dimension of features, and therefore, is computationally more expensive than PCA or LBP. A future study will be to reduce the feature dimension of the proposed method in an intelligent way.

\section{ACKNOWLEDGMENT}

This work is supported by the National Plan for Science and Technology, King Saud University, Riyadh, Saudi Arabia under project number 10-INF1044-02.

\section{REFERENCES}

[1] Ahonen, T., Hadid, A., Pietikäinen, M., "Face Description with Local Binary Patterns: Application to Face Recognition", IEEE T rans. Pattern Analysis and Machine Intelligence, 28 (12), pp. 2037-2041, 2006.

[2] Mäkinen, E., Raisamo, R., "An experimental comparison of gender classification methods", Pattern Recognition Letters. 29 (10), pp. 1544 1556,2008 .

[3] Veropoulos, K., Bebis, G., Webster, M., "Investigating the impact of face categorization on recognition performance", The First international conference on Advances in Visual Computing (ISVC'05), pp. 207-218. Lake Tahoe, 2005.

[4] Phillips, P.J., Hyeonjoon, M., Rizvi, S.A., Rauss, P.J., "The FERET evaluation methodology for face-recognition algorithms", IEEE Trans. on Pattern Analysis and Machine Intell., 22(10), pp. 1090-1104, 2000.

[5] Fang, Y., Wang, Z., "Improving LBP features for gender classification", International Conference on Wavelet Analysis and Pattern Recognition (ICW APR '08), pp. 373-377, Hong Kong, 2008.

[6] Ullah, I., Hussain, M., Muhammad, G., Aboalsamh, H., Bebis, G., Mirza, A., "Gender recognition from face images with local WLD descriptor", 19th International Conference on Systems, Signals and Image Processing (IWSSIP), pp. 417-420, Vienna, 2012.

[7] Ozbudak, O., Tukel, M., Seker, S., "Fast Gender Classification", IEEE International Conference on Computational Intelligence and Computing Research (ICCIC), pp. 1-5, Coimbatore, 2010.

[8] Shobeirinejad, A., Gao, Y., "Gender Classification Using Interlaced Derivative Patterns", 20th International Conference on Pattern Recognition (ICPR'10), pp. 1509-1512. Brisbane, 2010.

[9] Bui, L., Tran, D., Huang, X., Chetty, G., "Classification of gender and face based on gradient faces", European Workshop on Visual Information Processing (EUVIP'11), pp. 269-272, Paris, 2011.

[10] Mallat, S., A wavelet Tour of Signal Processing, The Sparse Way, Third Edition, Associated Press, pp. 631-637, MA, 2009.

[11] Qu, X., Yan, J., Xie, G., Zhu, Z., Chen, B., "A novel image fusion algorithm based on bandelet transform", Chinese Optics Letters, 5 (10), pp. 569-572, 2007.

[12] Zang, J., Lu, B.L., "A support vector machine classifier with automatic confidence and its application to gender classification", Neurocomputing. 74, pp. 1926-1935, 2011.

[13] Alexandre, L.A., "Gender recognition: A multiscale decision fusion approach”, Pattern Recognition Letters, 31, pp. 1422-1427, 2010. 